\title{
Tendencias en el consumo de tabaco adolescente en España (2002-2018)
}

\section{Trends in tobacco use among adolescents in Spain (2002-2018)}

\author{
Eva Leal-López*, Inmaculada Sánchez-Queija*, Garmen Moreno*. \\ * Departamento de Psicología Evolutiva y de la Educación. Universidad de Sevilla, Sevilla, España.
}

\section{Resumen}

El tabaco es la principal causa de muerte prevenible en todo el mundo. La adolescencia es una etapa clave en la iniciación al hábito tabáquico y en la proclividad a desarrollar adicción a esta sustancia. El objetivo de este trabajo es analizar cómo ha evolucionado el consumo de tabaco de los chicos y chicas adolescentes escolarizados en España desde 2002 a 2018 y si hay diferencias en las tendencias por sexo y por edad. La muestra está conformada por 51.046 participantes de 15 a 18 años, representativos de la población adolescente escolarizada en España en los años 2002, 2006, 2010, 2014 y 2018. Se utilizó el cuestionario de consumo de tabaco consensuado por el equipo internacional del estudio Health Behaviour in School-aged Children (HBSC). Se estimaron las Odds Ratio y los intervalos de confianza del 95\% mediante regresiones logísticas. Los resultados muestran una disminución en el consumo diario de tabaco adolescente entre 2002 $(26,5 \%)$ y $2018(8,7 \%)$ aunque con un periodo de estabilidad entre $2006(17,9 \%)$ y $2010(17,4 \%)$. Esta tendencia de descenso es mayor en las chicas (21,9 puntos) que en los chicos (13,1 puntos) hasta el punto de que en 2018 no hay diferencias en función del sexo. También es mayor en el grupo de 17-18 años (20,2 puntos) que en el de 15-16 años ( 15,8 puntos), aunque en este caso, permanecen las diferencias en función de la edad. La prevalencia de consumo de tabaco diario en adolescentes de 15 a 18 años es del 8,7\% en 2018. Se concluye la conveniencia de no suprimir ni disminuir las políticas de prevención y control del tabaquismo y se proponen nuevas medidas para hacer frente al problema de salud pública que está suponiendo el consumo de tabaco en España.

Palabras clave: Tabaco; Adolescencia; Análisis de tendencias;

Monitorización; Epidemiología.

\begin{abstract}
Smoking is the single greatest preventable cause of death in the world today. Adolescence is the developmental period during which smoking is most commonly initiated and addiction is likely to happen. The aim of this study is to examine trends in tobacco use among school-aged adolescents in Spain from 2002 to 2018 by sex and age. The sample is composed of 51,046 adolescents aged 15 to 18 . Data is representative of the adolescent school population in Spain in 2002, 2006, 2010, 2014 and 2018. The smoking questionnaire provided by the international team of the study Health Behavior in School-aged Children (HBSC) was used. Odds Ratios and 95\% confidence intervals were estimated using logistic regression. Data show a decrease in daily tobacco use between $2002(26.5 \%)$ and 2018 (8.7\%), but no change was found between 2006 (17.9\%) and 2010 (17.4\%). This decreasing pattern is stronger in girls than boys to the extent that no differences by sex were found in 2018. Similarly, the decrease was greater in older adolescents, but in this case, the differences by age remained. Daily smoking prevalence among Spanish adolescents aged 15 to 18 in 2018 is $8.7 \%$. Results confirm the need to maintain tobacco prevention and control policies. Measures are presented in order to fight this public health problem.

Key Words: Tobacco; Adolescence; Trends; Monitoring; Epidemiology.
\end{abstract}

Recibido: Enero 2018; Aceptado: Diciembre 2018.

Enviar correspondencia a:

Inmaculada Sánchez-Queija. Dirección postal: Departamento de Psicología Evolutiva y de la Educación (Universidad de Sevilla).

C/ Camilo José Cela, s/n. 41018. Sevilla. Tlfno: 955420 527. Fax: 954559544

E-mail: queija@us.es. 


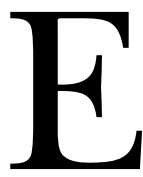
1 tabaco mata cada año alrededor de 7 millones de personas en el mundo (World Health Organization, 2017) y se estima que esta tasa de mortalidad ascenderá a 8,3 millones en 2030, siendo responsable del $10 \%$ de todas las muertes (Mathers y Loncar, 2006). En España, se produjeron más de un cuarto de millón de muertes atribuibles al tabaco entre 2010 y 2014 , lo que se corresponde con una media anual estimada de casi 52.000 muertes. Esta cifra supone el 13\% del total de las defunciones de personas mayores de 35 años en nuestro país (Ministerio de Sanidad, Servicios Sociales e Igualdad, 2016).

La adolescencia es un período crucial para la experimentación con las drogas y para el inicio de los trastornos adictivos. El desarrollo cerebral en estos años comprende cambios en su funcionalidad y organización que resultan en una mayor actividad de las áreas relacionadas con la búsqueda de experiencias nuevas, a la vez que el sistema de control inhibitorio se encuentra aún en crecimiento, lo que predispone a los chicos y chicas de estas edades, a una mayor realización de acciones impulsivas y conductas de riesgo, entre las que se incluye el consumo de drogas (Chambers, Taylor y Potenza, 2003).

Con relación al tabaco, las encuestas tanto nacionales (ESTUDES, EDADES) como internacionales (ESPAD, Eurobarómetro), sitúan la edad de inicio en el hábito tabáquico en la adolescencia y primeros años de juventud (European Commission, 2017; European Monitoring Centre for Drugs and Drug Addiction, 2016; Moreno et al., 2016; Plan Nacional sobre Drogas, 2018a; Plan Nacional sobre Drogas, 2018b). Las chicas y los chicos adolescentes que se inician en el consumo creen que podrán dejar de fumar pronto y de manera fácil, pero la naturaleza adictiva de la nicotina provoca que la mayoría desarrollen una adicción a esta sustancia y sigan fumando durante muchos años (Gruber, 2001). Además de los problemas de adicción al tabaco, fumar en la adolescencia tiene consecuencias inmediatas en la salud física (US Department of Health and Human Services, 2012), se relaciona con síntomas depresivos (Espada, Sussman, Huedo y Alfonso, 2011), ideación suicida (Bousoño et al., 2017) y con otras conductas adictivas como el consumo intensivo de alcohol (Golpe, Isorna, Barreiro, Braña y Rial, 2017), el consumo de cannabis o el juego (Míguez y Becoña, 2015). Ante esta realidad, las instituciones internacionales y los propios países tomaron medidas para luchar contra dicha problemática (Parlamento Europeo y del Consejo, 2014; WHO, 2003). En nuestro país, la más importante fue la aprobación en 2005 de la ley de medidas frente al tabaquismo (Ley 28, 2005) y su posterior modificación en 2010 (Ley 42, 2010), así como la puesta en marcha de proyectos de investigación y campañas de prevención (Ministerio de Sanidad, Servicios Sociales e Igualdad, 2017; Plan Nacional sobre Drogas, 2017).
Las medidas de control del tabaquismo parecen haber surtido efecto en la prevalencia de consumo de tabaco en la población adolescente mundial (European Monitoring Centre for Drugs and Drug Adiction, 2016; Hublet et al., 2015; Organisation for Economic Cooperation and Development, 2017;) y española (Plan Nacional sobre Drogas, 2018b; Villalbi et al., 2012), produciendo un descenso generalizado en el consumo, siguiendo así la misma tendencia que la población general (European Comission, 2017; Instituto Nacional de Estadística, 2013; Instituto Nacional de Estadística, 2017; Plan Nacional sobre Drogas, 2018a). Sin embargo, tal descenso no es continuo, ya que cuando se analizan más en profundidad los datos de los últimos años, se percibe cierta inestabilidad y algunas oscilaciones.

Analizando la encuesta ESTUDES entre los años 2002 y 2016 (Plan Nacional sobre Drogas, 2018b), se observan ciertas fluctuaciones en todos los tipos de consumo (diario, últimos 30 días, últimos 12 meses y alguna vez en la vida). Tomando específicamente los datos de consumo diario, dicho porcentaje presenta periodos de ligeros aumentos (2002-2004 y 2010-2012), de estabilidad (20062008 y 2014-2016) y de descenso (2004-2006, 2008-2010 y 2012-2014), situándose en 8,8\% en 2016. Las diferencias entre sexos casi han desaparecido $(8,6 \%$ en chicos y $9,0 \%$ en chicas) si se compara con 2002, donde la diferencia era de 6,5 puntos $(17,7 \%$ en chicos y $24,2 \%$ en chicas $)$. Otros estudios confirman estas irregularidades en la tendencia de consumo de tabaco adolescente en nuestro país para el mismo período (Villalbí et al., 2012).

A nivel internacional, la encuesta ESPAD (European Monitoring Centre for Drugs and Drug Adiction, 2016) también encuentra dicha tendencia descendiente de manera generalizada en todos los países europeos entre 2002 y 2010. El porcentaje de adolescentes que fuman a diario en 2015 fue del 10\%, encontrándose los valores más altos (20\% o más) en Bulgaria, Croacia, Italia y Rumania, mientras que los valores más bajos ( $5 \%$ o menos) se observaron en Albania, Islandia, Moldavia y Noruega. Las diferencias entre chicos y chicas han ido decreciendo con el tiempo hasta el punto de que en 2015 ya no existen o son mínimas. En la misma línea, el estudio internacional HBSC (Hublet et al., 2015) indica una reducción global del consumo entre 2002 y 2010, aunque encuentran tendencias diferentes según la región. Así, se observó un descenso en países del sur de Europa y anglosajones, estabilidad en países del norte y centroeuropeos, mientras que en los países del este de Europa y en Norteamérica se produjo un incremento en el consumo de tabaco adolescente. Estas fluctuaciones también se han visto reflejadas en el último Eurobarómetro, donde se ha detectado un aumento del consumo en el grupo más joven (15-24 años) pasando del 24\% en 2014 al 29\% en 2017 (European Comission, 2017). 
Ante estas circunstancias de inestabilidad observada en los informes sobre tabaco, se hace imprescindible realizar análisis más exhaustivos que permitan determinar cómo ha evolucionado realmente el tabaquismo adolescente en nuestro país en lo que llevamos de siglo y si esta tendencia se desarrolla de manera análoga en ambos sexos y en las distintas etapas de la adolescencia, o si, por el contrario, hay grupos que están mostrando patrones de consumo diferentes. Sólo de este modo se podrán tomar decisiones políticas ajustadas a la realidad de los datos. Por todo ello, el objetivo del presente trabajo es examinar las tendencias en el consumo de tabaco en una muestra de adolescentes representativa de la población escolarizada en España desde 2002 a 2018, analizando dichas tendencias por sexo y por edad.

\section{Métodos}

\section{Diseño y muestra del estudio}

Los datos de esta investigación forman parte del estudio Health Behaviour in School-aged Children (HBSC), cuyo objetivo es obtener una visión global de los estilos de vida de la población adolescente escolarizada que posibilite una promoción de la salud basada en la evidencia.

La muestra fue seleccionada siguiendo un muestreo aleatorio polietápico estratificado por conglomerados, siendo estratificada por edad, zona geográfica, hábitat y titularidad de centro educativo. Las unidades muestrales fueron las aulas, elegidas al azar del censo cedido por el Ministerio de Educación. Las tasas de respuesta al ítem para cada una de las ediciones contempladas en este estudio fueron del $98,52 \%$ en $2002,98,50 \%$ en $2006,96,57 \%$ en $2010,97,6 \%$ en 2014 y $95,73 \%$ en 2018 . En todos los análisis se han empleado pesos muestrales por edad, zona geográfica y titularidad del centro educativo para ajustar las descompensaciones de la muestra, ajustando así los datos a los parámetros poblacionales. Debido al escaso nivel de consumo de tabaco mostrado por las chicas y los chicos menores de 15 años, el análisis de este trabajo se centró en adolescentes de 15 a 18 años. El total de la muestra ascendió a 51.046 adolescentes con un error de precisión muestral estimado en 1,1\% aproximadamente en cada edición. Los datos son representativos del conjunto de escolares entre 15 y 18 años en España. Para más información técnica ver los informes del estudio HBSC para las distintas ediciones del estudio (Moreno, Muñoz, Pérez y Sánchez-Queija, 2005; Moreno et al., 2008; Moreno et al., 2012; Moreno et al., 2016; Moreno et al., en prensa).

\section{Procedimiento e instrumento}

Los datos fueron recogidos mediante cuestionarios completados por el alumnado en horario escolar y con garantías totales de anonimato, como dictan las directrices del protocolo internacional del estudio (Roberts et al., 2009).
De este cuestionario, aprobado por el Comité Coordinador de Ética de la Investigación Biomédica de Andalucía, se seleccionaron las variables sexo (chico / chica), edad (15-16 años / 17-18 años), edición (2002 / $2006 / 2010$ / 2014 / 2018) y frecuencia de consumo de tabaco. Dicha frecuencia se evaluó con la pregunta: ¿Con qué frecuencia fumas tabaco en la actualidad? Las categorías de respuesta fueron: todos los días / al menos una vez a la semana, pero no todos los días / menos de una vez a la semana / no fumo.

\section{Análisis estadístico}

Los resultados se presentan desagregados por sexo y edad. En cuanto a la edad, se presentan en dos grupos. Por un lado, el grupo de 15-16 años que permanecen escolarizados obligatoriamente. Por otro, el grupo de 17-18 años que continúan en el sistema educativo de manera voluntaria. Los datos se analizaron con regresión logística, método utilizado previamente en otros estudios de tendencias nacionales e internaciones (Hublet, et al., 2006; Looze, et al., 2013; Sánchez-Queija, García-Moya y Moreno, 2017; Sánchez-Queija, Moreno, Rivera y Ramos, 2015; Schnohr at al., 2015; Zaborskis, Sumskas, Maser y Pudule, 2006). Para ello se calcularon las Odds Ratio (OR) y los intervalos de confianza al 95\% (IC95\%). En primer lugar, se realizaron ecuaciones de regresión logística para conocer las diferencias en función del sexo y los grupos etarios en cada una de las ediciones contempladas (2002, 2006, 2010, 2014 y 2018). En segundo lugar, se llevaron a cabo los análisis de tendencias. En estos análisis, se efectuó una comparación del periodo completo, es decir, entre 2002 y 2018, para poder obtener una visión global de la tendencia, y también se realizaron comparaciones cuatrienales para conocer más en profundidad dicha tendencia. Para ello, el 2002 será la referencia para el cambio entre 2002 y 2006; 2006 será la referencia para el cambio respecto a 2010; 2010 será la referencia frente a 2014; finalmente, 2014 será la referencia para el cambio respecto a 2018. Ambos análisis de tendencias se realizaron teniendo en cuenta las variables sexo y edad para conocer si los patrones de consumo de tabaco adolescente están evolucionando de manera similar o diferente para chicos y chicas y para el grupo de 15-16 años y el de 17-18 años. Para los análisis estadísticos se utilizó el programa estadístico SPSS v.21.

\section{Resultados}

La tabla 1 expone los datos de la muestra de este estudio.

En la tabla 2 se presentan las prevalencias de los distintos tipos de consumo por sexo y edad, excepto los de consumo diario que se presentarán más detalladamente en la tabla 3 y figura 1. Los datos muestran un descenso en términos generales tanto en el consumo semanal como en el de menos de una vez a la semana, así como un incremento 
Tabla 1. Descripción de la muestra de las cinco ediciones según edad y sexo.

\begin{tabular}{llcccccc}
\hline & & $\begin{array}{c}\mathbf{2 0 0 2} \\
\mathbf{n}\end{array}$ & $\begin{array}{c}\mathbf{2 0 0 6} \\
\mathbf{n}\end{array}$ & $\begin{array}{c}\mathbf{2 0 1 0} \\
\mathbf{n}\end{array}$ & $\begin{array}{c}\mathbf{2 0 1 4} \\
\mathbf{n}\end{array}$ & $\begin{array}{c}\mathbf{2 0 1 8} \\
\mathbf{n}\end{array}$ & $\begin{array}{c}\text { Total } \\
\mathbf{n}\end{array}$ \\
\hline \multirow{2}{*}{$\mathbf{1 5 - 1 6}$ años } & Chicos & 1374 & 2032 & 1237 & 2949 & 3655 & 11247 \\
& Chicas & 1531 & 2231 & 1350 & 3082 & 3619 & 11813 \\
\multirow{2}{*}{$\mathbf{1 7 - 1 8}$ años } & Chicos & 1454 & 2356 & 1007 & 3909 & 4824 & 13550 \\
& Chicas & 1643 & 3115 & 975 & 3800 & 4903 & 14436 \\
\hline Total & & 6002 & 9734 & 4569 & 13740 & 17001 & 51046 \\
\hline
\end{tabular}

Tabla 2. Prevalencia de consumo de tabaco semanal, menos de una vez a la semana y de no consumo en la población adolescente escolarizada en España en 2002, 2006, 2010, 2014 y 2018.

\begin{tabular}{|c|c|c|c|c|c|c|c|c|c|c|c|c|}
\hline \multirow[t]{2}{*}{ Tipos de consumo } & \multirow[t]{2}{*}{ Edad (años) } & \multirow[t]{2}{*}{ Sexo } & \multicolumn{2}{|c|}{2002} & \multicolumn{2}{|c|}{2006} & \multicolumn{2}{|r|}{2010} & \multicolumn{2}{|c|}{2014} & \multicolumn{2}{|c|}{2018} \\
\hline & & & $\%$ & IC & $\%$ & IC & $\%$ & IC & $\%$ & IC & $\%$ & IC \\
\hline \multirow{4}{*}{ Semanal } & \multirow{2}{*}{$15-16$} & Chicos & 7,1 & $6,4-7,7$ & 4,6 & $4,1-5,0$ & 3,7 & $3,1-4,2$ & 4,1 & $3,7-4,4$ & 3,9 & $3,6-4,1$ \\
\hline & & Chicas & 8,3 & $7,6-8,9$ & 7,3 & $6,7-7,8$ & 6,1 & $5,4-6,7$ & 5,6 & $5,2-5,9$ & 5,0 & $4,6-5,3$ \\
\hline & \multirow{2}{*}{$17-18$} & Chicos & 7,3 & $6,6-7,9$ & 5,1 & $4,6-5,5$ & 7,6 & $6,8-8,3$ & 6,2 & $5,7-6,6$ & 6,1 & $5,7-6,4$ \\
\hline & & Chicas & 6,9 & $6,2-7,5$ & 7,1 & $6,5-7,6$ & 7,7 & $6,9-8,4$ & 6,5 & $6,0-6,9$ & 7,2 & $6,8-7,5$ \\
\hline \multirow{4}{*}{$\begin{array}{l}\text { Menos de una vez } \\
\text { a la semana }\end{array}$} & \multirow{2}{*}{$15-16$} & Chicos & 7,7 & $7,0-8,3$ & 5,1 & $4,6-5,5$ & 4,9 & $4,2-5,5$ & 3,8 & $3,4-4,1$ & 5,2 & $4,8-5,5$ \\
\hline & & Chicas & 5,6 & $5,0-6,1$ & 7,6 & $7,0-8,1$ & 6,7 & $5,9-7,4$ & 4,8 & $4,4-5,1$ & 6,8 & $6,4-7,1$ \\
\hline & \multirow{2}{*}{$17-18$} & Chicos & 6,2 & $5,5-6,8$ & 5,5 & $5,0-5,9$ & 5,6 & $4,9-6,2$ & 6,7 & $6,2-7,1$ & 5,2 & $4,8-5,5$ \\
\hline & & Chicas & 6,0 & $5,3-6,6$ & 6,2 & $5,7-6,6$ & 6,6 & $5,8-7,3$ & 5,5 & $5,1-5,8$ & 6,8 & $6,4-7,1$ \\
\hline \multirow{3}{*}{ No consumo } & $15-16$ & Chicas & 62,1 & $60,8-63,3$ & 69,8 & $68,8-70,7$ & 70,0 & $68,6-71,3$ & 82,8 & $82,1-83,4$ & 82,4 & $81,8-82,9$ \\
\hline & \multirow{2}{*}{$17-18$} & Chicos & 61,3 & $60,0-62,5$ & 71,5 & $70,6-72,3$ & 66,2 & $64,8-67,5$ & 75,1 & $74,3-75,8$ & 77,2 & $76,5-77,8$ \\
\hline & & Chicas & 50,5 & $49,2-51,7$ & 62,5 & $61,5-63,4$ & 64,3 & $62,9-65,6$ & 73,5 & $72,7-74,2$ & 75,2 & $74,5-75,8$ \\
\hline
\end{tabular}

de adolescentes que no fuman entre 2002 y 2018, aunque con algún pico de consumo, especialmente entre las ediciones 2006 y 2010.

Para analizar en profundidad el consumo diario, la variable se dicotomizó en consumo diario (codificado 1) frente al resto de consumos y no consumo (codificado 0 ). En la tabla 3 se presentan los resultados de la regresión logística entre la variable consumo diario y las variables sexo, edad y combinación de sexo-edad en cada una de las ediciones analizadas en este trabajo. Se observa que el porcentaje de adolescentes que fuman diariamente ha pasado de un 26,5\% en 2002 a un 8,7\% en 2018. Respecto al sexo, las chicas de ambos grupos etarios fumaban diariamente en mayor proporción que los chicos en 2002 y 2006. Sin embargo, en 2010 y 2014 estas diferencias disminuyeron, siendo significativas únicamente en uno de los dos grupos (15-16 años en 2010 y 17-18 años en 2014) y en 2018 no se encontraron diferencias significativas en ninguno de los grupos de edad. En cuanto a la edad, el grupo de 17-18 años presenta mayor consumo que el de 15-16 años en to- das las ediciones del estudio y en ambos sexos. En la figura 1 puede observarse cómo ha evolucionado el consumo de tabaco en cada grupo específico en estos 16 años.

En la tabla 4 se muestran los análisis de tendencias, tanto para la comparación del período completo (2002 vs 2018), como para las comparaciones cuatrienales (2002 vs 2006, 2006 vs 2010,2010 vs 2014 y 2014 vs 2018). Al analizar la tendencia global, se confirma la disminución del consumo de tabaco diario adolescente, pasando de un $26,5 \%$ al inicio del período estudiado, a un $8,7 \%$ al final del período $(\mathrm{OR}=0,22 ; 95 \% \mathrm{CI}=0,20-0,25)$. Analizando la evolución en cada grupo específico se detecta un fuerte descenso en ambos grupos de chicas, especialmente en las de mayor edad $(25,1$ puntos $)(\mathrm{OR}=0,21 ; 95 \% \mathrm{CI}=0,18-0,24)$. No obstante, al comparar cada edición tomando como referencia la edición anterior, se percibe un periodo de estabilidad entre 2006 y 2010 para ambos sexos y en todas las edades.

Para analizar si existen diferencias estadísticamente significativas en la tendencia de consumo diario de tabaco entre chicos y chicas, se analizó la interacción entre el sexo y 
Tabla 3. Porcentaje de consumo diario de tabaco, OR y IC95\% en la población adolescente escolarizada en España en 2002, 2006, 2010, 2014 y 2018.

\begin{tabular}{|c|c|c|c|c|c|}
\hline & 2002 & 2006 & 2010 & 2014 & 2018 \\
\hline$\%$ total & 26,5 & 17,9 & 17,4 & 10,3 & 8,7 \\
\hline \multicolumn{6}{|l|}{ Sexo } \\
\hline Chicos (\%) & 21,9 & 14,6 & 15,7 & 9,5 & 8,8 \\
\hline Chicas (\%) & 30,5 & 20,6 & 19,0 & 11,1 & 8,6 \\
\hline OR $(95 \% \mathrm{Cl})$ & $1,560(1,387-1,754)^{\star \star \star}$ & $1,511(1,357-1,683)^{\star \star \star}$ & $1,260(1,080-1,469)^{\star \star}$ & $1,190(1,064-1,331)^{\star \star}$ & $0,973(0,873-1,085)$ \\
\hline \multicolumn{6}{|l|}{ Edad } \\
\hline $15-16(\%)$ & 21,3 & 13,2 & 14,6 & 6,5 & 5,5 \\
\hline $17-18(\%)$ & 31,3 & 21,5 & 21,1 & 13,3 & 11,1 \\
\hline OR $(95 \% \mathrm{Cl})$ & $1,679(1,493-1,888)^{\star \star \star}$ & $1,805(1,615-2,016)^{\star \star \star}$ & $1,566(1,343-1,827)^{\star \star \star}$ & $2,187(1,934-2,473)^{\star \star \star}$ & $2,155(1,908-2,433)^{\star \star \star \star}$ \\
\hline \multicolumn{6}{|l|}{ Por grupos } \\
\hline Chicos 15-16 (\%) & 18,4 & 10,8 & 11,7 & 6,2 & 5,3 \\
\hline Chicas 15-16 (\%) & 23,9 & 15,4 & 17,2 & 6,8 & 5,7 \\
\hline OR $(95 \% \mathrm{Cl})$ & $1,390(1,160-1,666)^{\star \star \star}$ & $1,505(1,254-1,807)^{\star \star \star}$ & $1,579(1,262-1,976)^{\star \star \star}$ & $\begin{array}{l}1,107 \\
(0,900-1,362)\end{array}$ & $\begin{array}{l}1,089 \\
(0,886-1,338)\end{array}$ \\
\hline Chicos 17-18 (\%) & 25,2 & 17,9 & 20,7 & 12,0 & 11,5 \\
\hline Chicas 17-18 (\%) & 36,6 & 24,2 & 21,5 & 14,6 & 10,8 \\
\hline OR $(95 \% \mathrm{Cl})$ & $1,711(1,464-1,999)^{\star \star \star}$ & $1,464(1,280-1,675)^{\star \star \star}$ & $1,047(0,843-1,299)$ & $1,256(1,009-1,435)^{\star \star}$ & $\begin{array}{l}0,927 \\
(0,815-1,055)\end{array}$ \\
\hline
\end{tabular}

Nota. ${ }^{*} p<.05 ;{ }^{* \star} p<.01 ;{ }^{\star \star \star} p<.001$

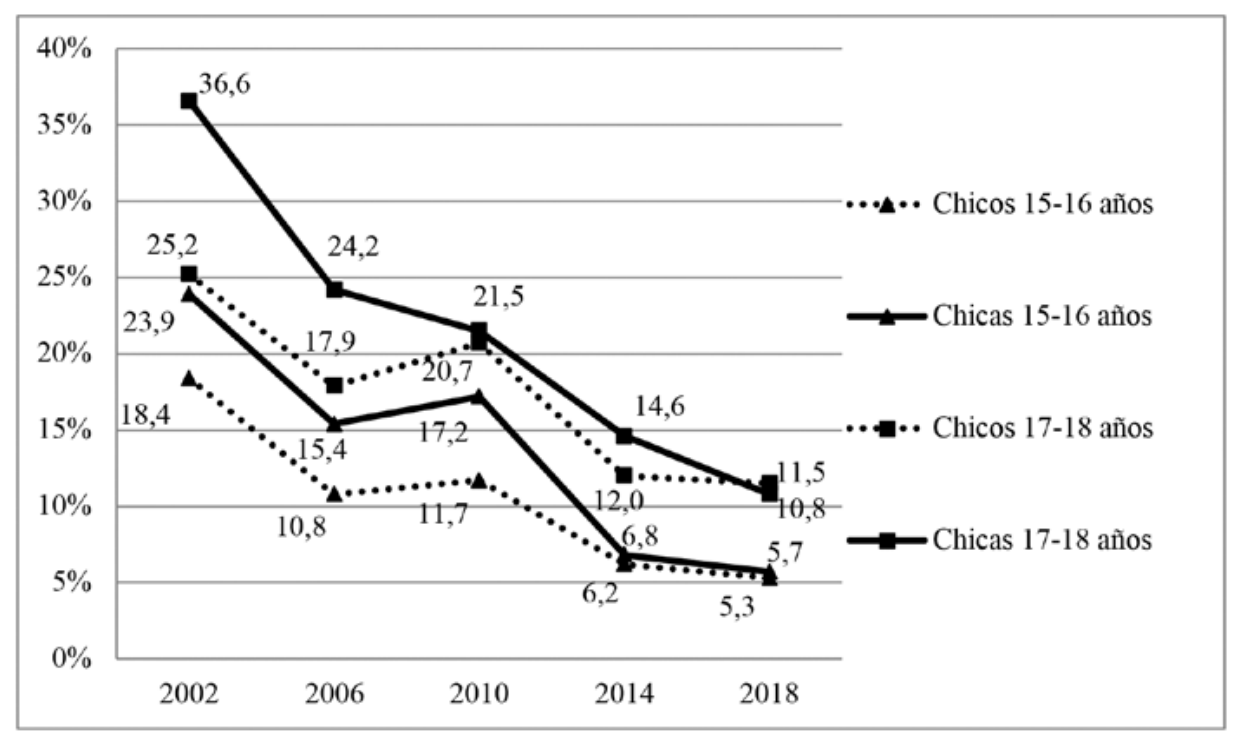

Figura 1. Consumo diario de tabaco en función del sexo y la edad.

las ediciones del estudio (tabla 4). Los datos muestran que sí existe interacción estadísticamente significativa entre la edición y el sexo en el período completo entre 2002 y 2018 $(\mathrm{OR}=1,61 ; 95 \% \mathrm{CI}=1,37-1,90)$, siendo el descenso en las chicas (21,9 puntos) más pronunciado que en los chicos (13,1 puntos). En la misma línea, se analizó la interacción entre los grupos de edad y las ediciones del estudio para conocer si el descenso en el consumo a lo largo de los años era mayor en un grupo etario que en el otro. Los resultaron muestran que la disminución en el consumo es mayor entre el grupo de 17-18 años (20,2 puntos) que entre el de $15-16$ años (15,8 puntos) $(\mathrm{OR}=0,78 ; 95 \% \mathrm{CI}=0,66-0,93)$. 
Tabla 4. Consumo diario de tabaco en la población adolescente escolarizada en España: OR (IC 95\%) de las variables edición, sexo, edad, las interacciones de sexo-edición y edad-edición, asi como de cada grupo específico para la comparación del periodo completo (2002 vs 2018) y las comparaciones entre ediciones (2002 vs 2006, 2006 vs 2010, 2010 vs 2014 y 2014 vs 2018).

\begin{tabular}{|c|c|c|c|c|c|}
\hline \multicolumn{6}{|l|}{ OR (IC 95\%) } \\
\hline & 2002 vs 2018 & 2002 vs 2006 & 2006 vs 2010 & 2010 vs 2014 & 2014 vs 2018 \\
\hline Edición & $0,222(0,196-0,252)^{\star \star \star}$ & $0,578(0,512-0,651)^{\star \star \star}$ & $0,937(0,806-1,091)$ & $0,557(0,477-0,651)^{\star \star \star *}$ & $0,736(0,653-0,830)^{\star * \star}$ \\
\hline Sexo & $1,567(1,393-1,764)^{\star \star \star}$ & $1,567(1,393-1,764)^{\star \star \star}$ & $1,479(1,327-1,648)^{\star \star \star}$ & $1,279(1,095-1,492)^{\star \star}$ & $1,210(1,082-1,354)^{\star \star}$ \\
\hline Edad & $1,686(1,499-1,897)^{\star \star \star}$ & $1,686(1,499-1,897)^{\star \star \star}$ & $1,777(1,591-1,986)^{\star \star \star}$ & $1,579(1,354-1,842)^{\star \star \star}$ & $2,197(1,942-2,484)^{\star \star \star}$ \\
\hline Sexo-edición & $1,615(1,375-1,897)^{\star \star}$ & $1,060(0,903-1,244)$ & $1,156(0,957-1,397)$ & $1,056(0,873-1,279)$ & $1,248(1,067-1,459)^{\star *}$ \\
\hline Edad-edición & $0,782(0,661-0,927)^{\star \star}$ & $0,949(0,807-1,116)$ & $1,126(0,931-1,361)$ & $0,719(0,590-0,875)^{\star \star}$ & $1,019(857-1,212)$ \\
\hline Chicos 15-16 & $0,247(0,202-0,302)^{\star \star \star}$ & $0,536(0,440-0,652)^{\star \star \star}$ & $1,090(0,872-1,364)$ & $0,503(0,399-0,633)^{\star \star \star}$ & $0,841(0,680-1,040)$ \\
\hline Chicas 15-16 & $0,193(0,161-0,233)^{\star \star \star}$ & $0,580(0,492-0,684)^{\star \star \star}$ & $1,144(0,953-1,374)$ & $0,352(0,289-0,430)^{\star \star \star}$ & $0,827(0,677-1,012)$ \\
\hline Chicos 17-18 & $0,386(0,333-0,448)^{\star \star *}$ & $0,648(0,522-0,760)^{\star \star \star}$ & $1,194(0,992-1,438)$ & $0,521(0,435-0,625)^{\star \star \star}$ & $0,958(0,839-1,094)$ \\
\hline Chicas 17-18 & $0,209(0,183-0,240)^{\star \star \star}$ & $0,555(0,487-0,632)^{\star \star \star}$ & $0,854(0,717-1,016)$ & $0,625(0,523-0,747)^{\star \star \star}$ & $0,707(0,621-0,805)^{\star \star *}$ \\
\hline
\end{tabular}

Nota. Las categorías de referencias para la variable edición son: 2002 para la comparación 2002 vS 2018; 2002 para la comparación 2002 vs 2006 ; 2006 para la com paración 2006 vs 2010; 2010 para la comparación 2010 vs 2014; 2014 para la comparación 2014 vs 2018. Para la variable sexo la categoría de referencia es ‘chico’ y para la variable edad es '15-16 años'. ${ }^{\star} p<.05 ;{ }^{* \star} p<.01 ;{ }^{* \star *} p<.001$

\section{Discusión}

Este trabajo se centra en el análisis de las tendencias de consumo de tabaco adolescente en lo que llevamos de siglo. Los resultados indican un descenso en términos absolutos entre 2002 y 2018 en ambos sexos y en todas las edades, lo que coincide con otros estudios nacionales (Plan Nacional sobre Drogas, 2018b; Villalbi et al., 2012) e internacionales (European Monitoring Centre for Drugs and Drug Adiction, 2016; Hublet et al., 2015; Organisation for Economic Cooperation and Development, 2017). No obstante, los datos indican que esta tendencia no es continua, sino que se alterna con un periodo en el que el consumo se mantiene estable (2006-2010). De hecho, en 3 de los 4 grupos analizados el porcentaje de adolescentes que fuman diariamente crece entre 2006 y 2010, aunque tales diferencias no llegan a ser estadísticamente significativas. Esta inestabilidad en la tendencia del consumo coincide con la detectada por la encuesta ESTUDES (Plan Nacional sobre Drogas, 2018b) y por Villalbi et al. (2012).

En cuanto a los patrones de consumo por sexo y edad, los resultados de este trabajo muestran que la tendencia de descenso en las chicas es mayor que en los chicos, lo que ha producido que las diferencias de sexos hayan desaparecido en 2018. De esta forma, se ha revertido la tendencia ascendente que se venía observado anteriormente, donde hubo momentos en los que las chicas fumadoras, especialmente las mayores, mostraban un consumo muy superior al de los chicos (Mendoza, López y Sagrera, 2007). Del mismo modo, la disminución en el consumo diario de tabaco es más pronunciada en el grupo de mayor edad en comparación con el de menor edad, aunque en este caso, las diferencias siguen manteniéndose. Estos datos ratifican y complementan las tendencias observadas en otros estu- dios nacionales e internacionales sobre tabaco adolescente (Díaz Geada, Busto Miramontes y Caamaño Isorna, 2018; European Monitoring Centre for Drugs and Drug Adiction, 2016; Hublet et al., 2015; Plan Nacional sobre Drogas, 2018b).

Si bien se ha producido un importante descenso en el consumo diario de tabaco adolescente en España, mostrando en 2015 (que es el último año en que existen valores internacionales de comparación) unos valores similares a la media europea, hay que resaltar también que la prevalencia de chicos y chicas que fuman a diario $(8,7 \%)$ sigue siendo más alta de lo deseable y mayor que en otros países (European Monitoring Centre for Drugs and Drug Adiction, 2016). Así, los datos muestran que en 2018 el 5,5\% de adolescentes de 15-16 años fuman tabaco diariamente, ascendiendo a un 11,1\% a los 17-18 años. En este último caso, hay que destacar que se trata únicamente de adolescentes que aún permanecen en el sistema educativo, por lo que este porcentaje podría ser incluso mayor al tener en cuenta la población no escolarizada a estas edades.

Puesto que la adolescencia es una etapa clave para el inicio y mantenimiento del hábito tabáquico (Chambers et al., 2003; European Commission, 2017; European Monitoring Centre for Drugs and Drug Addiction, 2016; Gruber, 2001; Moreno et al., 2016; Plan Nacional sobre Drogas, 2018a; Plan Nacional sobre Drogas, 2018b), se hace imprescindible y prioritario desarrollar políticas de prevención y control del tabaquismo que focalicen en estas edades. Más aún si se tienen en cuenta los últimos datos de la Encuesta sobre Alcohol y otras Drogas en España (EDADES) presentada recientemente en nuestro país donde informan que el porcentaje de consumo diario en población de 15-64 años ha aumentado entre 2015 y 2017 (Plan Nacional sobre Drogas, 2018a). 
Evitar el inicio de quienes aún no han comenzado a fumar y lograr el abandono de quienes sí fuman en la adolescencia, evitaría más del $90 \%$ de la mortalidad por cáncer de pulmón atribuible al tabaco y reduciría el gasto público en salud por enfermedades relacionadas con el tabaco, el cual se sitúa entre el 6 y el $15 \%$ en los países en desarrollo (Jha, 2011). Sin embargo, en el ámbito estatal, los esfuerzos por luchar contra este problema de salud pública no parecen estar siendo suficientes en la actualidad: la Ley antitabaco no logró tener el impacto esperado (Grupo de trabajo sobre tabaquismo de la Sociedad Española de Epidemiología, 2017), cesaron los proyectos de investigación sobre el tabaco financiados por el Plan Nacional Sobre Drogas (Plan Nacional Sobre Drogas, 2017), se suprimieron las campañas de prevención nacionales específicas de tabaco (Ministerio de Sanidad, Servicios Sociales e Igualdad, 2017; Plan Nacional Sobre Drogas, 2017), se eliminó el Observatorio de Tabaquismo (Ley $N^{\circ} 15,2014$ ) y la transposición de la nueva Directiva Europea no se culminó como proponían los grupos expertos en el tema (Comité Nacional de Prevención del Tabaquismo, 2017).

La problemática del tabaquismo adolescente sigue presente, pero la sensibilización con el tema y las acciones para hacerle frente han ido desapareciendo con el tiempo. Nos encontramos en un momento clave en España, ya que recientemente se ha procedido a la modificación de la Ley 28/2005 para poder completar la transposición de la Directiva Europea 2014/40/UE, pero, sin embargo, las modificaciones efectuadas han sido mínimas y referidas exclusivamente a equiparar ciertas restricciones entre los productos de tabaco, con los cigarrillos electrónicos y envases de recarga. Se ha perdido una gran oportunidad de retomar la lucha contra esta epidemia que provoca miles de muertes al año en nuestro país y de poner en marcha una serie de medidas necesarias para mejorar el estado de salud de nuestros chicos y chicas adolescentes y de la población en general, como así vienen proponiendo varios grupos de trabajo especializados en la temática (Grupo de trabajo sobre tabaquismo de la Sociedad Española de Epidemiología, 2017; Comité Nacional de Prevención del Tabaquismo, 2017). Entre las medidas propuestas por estos grupos están las relacionadas con el empaquetado neutro; el control sobre la publicidad, promoción y patrocinio del tabaco; la ampliación de los espacios libres de humo; el establecimiento de impuestos sobre todos los productos del tabaco; la realización de programas de prevención, sensibilización, investigación y formación sobre el tabaquismo; así como una atención adecuada a las personas fumadoras. Y estas medidas no pueden quedarse en una mera declaración de intenciones. Su puesta en marcha requiere considerables esfuerzos por parte del Gobierno no sólo en la financiación y en la provisión de recursos, sino también en la firmeza de su aplicación ante las presiones de la industria tabacalera.
Este trabajo no está exento de limitaciones. La principal el ser un estudio transversal en el que los datos se recogen en un solo momento temporal y mediante autoinformes anónimos, por lo que existen ciertos riesgos como la deseabilidad social o la infravaloración de las conductas problemáticas. No obstante, este tipo de metodología es la que se utiliza con más frecuencia en estudios de esta tipología (Villalbí, Suelves, Saltó y Cabezas, 2011). La segunda limitación es que la muestra se limite a estudiantes, dejando fuera a los y las jóvenes de 17-18 años que abandonan la escolaridad, lo que puede estar sesgando los datos, muy probablemente infravalorando la proporción de tabaquismo adolescente. No obstante, el estudio posee importantes fortalezas. Sin duda alguna, el gran número de participantes en la muestra aporta un innegable valor descriptivo al trabajo y presenta una fotografía bastante precisa sobre el consumo de tabaco en estas edades. Asimismo, contar con cinco momentos temporales con los que poder llevar a cabo análisis estadísticos pertinentes para examinar las tendencias de consumo, permite conocer con bastante exactitud cómo ha ido evolucionando dicha fotografía a lo largo de estos 16 años y poder tomar decisiones políticas basadas en la evidencia científica. En conclusión, los resultados del presente estudio constituyen una valiosa contribución a la bibliografía sobre las tendencias del consumo de tabaco adolescente en el siglo XXI en nuestro país, así como a las políticas de prevención y control del tabaquismo.

\section{Reconocimientos}

El estudio HBSC en España se ha financiado gracias a sucesivos convenios de colaboración con el Ministerio de Sanidad, Servicios Sociales e Igualdad / Sanidad y Política Social / Sanidad, Política Social e Igualdad / Sanidad y Política Social / Sanidad y Consumo, y la Universidad de Sevilla. Códigos: 3153/0294; 2603/0294; 2315/0294; 2053/0294; 0551/0294/2010; 1298/0294; 0551/0294/2009; SI-039/08; SI-084/07; SI-080/06; SI150/05. Nuestro sincero agradecimiento a dicha institución, a todo el equipo HBSC España, así como a los centros escolares, profesorado y alumnado participante en las distintas ediciones del estudio. Este artículo es parte de la tesis doctoral de Eva Leal-López, en la Universidad de Sevilla.

\section{Conflicto de intereses}

Las personas autoras de este trabajo declaran que no existe conflicto de intereses.

\section{Referencias}

Bousoño, M., Al-Halabí, S., Burón, P., Garrido, M., Díaz-Mesa, E.M., Galván, G.,... Bobes, J. (2017). Uso y abuso de sustancias psicotrópicas e internet, psicopatología e 
ideación suicida en adolescentes. Adicciones, 29, 97-104. doi:10.20882/adicciones.29.2.

Chambers, R.A., Taylor, J.R. y Potenza, M.N. (2003). Developmental neurocircuitry of motivation in adolescence: a critical period of addiction vulnerability. American Journal of Psychiatry, 160, 1041-1052. doi:10.1176/appi. ajp.160.6.1041.

Comité Nacional de Prevención del Tabaquismo. (2017). Alegaciones al anteproyecto de ley por la que se modifica la Ley 28/2005. Recuperado de http://www.cnpt.es/documentacion/noticias/ ea562bfb797b847b592101b33a0fb3054b6705880e04ed36eb62f3ec931be641.pdf

Díaz Geada, A., Busto Miramontes, A. y Caamaño Isorna, F. (2018). Alcohol, tobacco and cannabis consumption in adolescents from a multicultural population (Burela, Lugo). Adicciones, 30, 264-270. doi:10.20882/adicciones.915.

Espada, J.P., Sussman, S., Huedo, T.B. y Alfonso, J.P. (2011). Relation between Substance Use and Depression among Spanish Adolescents. International Journal of Psychology and Psychological Therapy, 11, 79-90.

Golpe, S., Isorna, M., Barreiro, C., Braña, T. y Rial A. (2017). Binge drinking among adolescents: prevalence, risk practices and related variables. Adicciones, 29, 256267. doi:10.20882/adicciones.932.

European Commission. (2017). Special Eurobarometer 458 "Attitudes of Europeans towards tobacco and electronic cigarettes". Recuperado de https:/ / publications.europa.eu /en/publication-detail/-/publication/2f01a3d1-0a f2-11e8-966a-01aa75ed71a1/language-en.

European Monitoring Centre for Drugs and Drug Addiction [EMCDDA]. (2016). The European School Survey Project on Alcohol and Other Drugs (ESPAD) 1195- 2015. Luxemburgo: Publications Office of the European Union. Recuperado de http:/ / espad.org/report/home/

Gruber, J. (2001). Youth smoking in the 1990: why did it rise and what are the long-run implications. American Economic Review, 91, 85-90.

Grupo de Trabajo sobre Tabaquismo de la Sociedad Española de Epidemiología. (2017). Evaluación de las políticas de control del tabaquismo en España (Leyes 28/2005 y 42/2010). Revisión de la evidencia. Recuperado de http:/ / www.seepidemiologia.es/documents/dummy/V9.0\% 20-\%20Libro \% 20 Tabaquismo\%202017\%20-\%20Abierto\%20Final.pdf.

Hublet, A., Bendtsen, P., de Looze, M.E., Fotiou, A., Donnelly, P., Vilhjalmsson, R.,... ter Bogt, T.F. (2015). Trends in the co-occurrence of tobacco and cannabis use in 15-year-olds from 2002 to 2010 in 28 countries of Europe and North America. European Journal of Public Health, 25, 73-75. doi:10.1093/eurpub/ckv032.

Hublet, A., de Bacquer, D., Valimaa, R., Godeau, E., Schmid, H., Rahav, G. y Maes, L. (2006). Smoking trends among adolescents from 1990 to 2002 in ten European countries and Canada. BMC Public Health, 6, 280-286. doi:10.1186/1471-2458-6-280.
Instituto Nacional de Estadística [INE]. (2013). Encuesta Nacional de Salud 2011-2012 (ENSE). Madrid, España. Ministerio de Sanidad, Servicios Sociales e Igualdad. Recuperado de https://www.mscbs.gob.es/estadEstudios/ estadisticas/encuestaNacional/encuesta2011.htm.

Instituto Nacional de Estadística [INE]. (2017). Encuesta Europea de Salud 2014 (EESE). Madrid, España. Ministerio de Sanidad, Servicios Sociales e Igualdad. Recuperado de https://www.mscbs.gob.es/estadEstudios/estadisticas / EncuestaEuropea/Enc_Eur_Salud_en_Esp_2014.htm.

Jha, P. (2011). Avoidable deaths from smoking: a global perspective. Public Health Reviews, 33, 569-600. doi:10.1007/BF03391651.

Ley 15/2014, de 16 de septiembre, de racionalización del Sector Público y otras medidas de reforma administrativa. Boletín Oficial del Estado. Recuperado de https:/ / www.boe.es/boe/dias / 2014/09/17/pdfs / BOE-A-2014-9467.pdf.

Ley 28/2005, de 26 de diciembre, de medidas sanitarias frente al tabaquismo y reguladora de la venta, el suministro, el consumo y la publicidad de los productos del tabaco. Boletín Oficial del Estado. Recuperado de https:/ / boe.es/boe/dias/2005/12/27/pdfs/A42241-42250.pdf.

Ley $42 / 2010$, de 30 de diciembre, por la que se modifica la Ley 28/2005, de 26 de diciembre, de medidas sanitarias frente al tabaquismo y reguladora de la venta, el suministro, el consumo y la publicidad de los productos del tabaco. Recuperado de https:/ / www.boe.es/buscar/ pdf/2010/BOE-A-2010-20138-consolidado.pdf.

Looze, M., Ter Bogt, T., Hublet, A., Kuntsche, E., Richter, M., Zsiros, E.,... Vollebergh, W. (2013). Trends in educational differences in adolescent daily smoking across Europe, 2002-10. European Journal of Public Health, 23, 846-852. doi:10.1093/eurpub/ckt022.

Mathers, C.D. y Loncar, D. (2006). Projections of Global Mortality and Burden of Disease from 2002 to 2030. PLoS Medicine, 3 (11), 442. doi:10.1371/journal. pmed.0030442.

Mendoza, R., López, P. y Sagrera, M.R. (2007). Diferencias de género en la evolución del tabaquismo adolescente en España (1986-2002). Adicciones, 19, 273-288. doi:10.20882/adicciones.19.3.

Míguez, M.C. y Becoña, E. (2015). ¿El consumo de cigarrillos y alcohol se relaciona con el consume de cannabis y el juego problema en adolescentes españoles? Adicciones, 27, 8-16. doi:10.20882/adicciones.27.1.

Ministerio de Sanidad, Servicios Sociales e Igualdad. (2016). Muertes atribuibles al consumo de tabaco en España, 20002014. Madrid, España: Ministerio de Sanidad, Servicios Sociales e Igualdad. Recuperado de https://www.mscbs. gob.es/estadEstudios/estadisticas/estadisticas/estMinisterio/mortalidad/docs/MuertesTabacoEspana2014.pdf.

Ministerio de Sanidad, Servicios Sociales e Igualdad. (2017). Campañas estatales de prevención de tabaquismo. 
Recuperado de http://www.msssi.gob.es/ciudadanos/ proteccionSalud/tabaco/campana_Estatal.htm.

Moreno, M.C., Muñoz, M.V., Pérez, P. y Sánchez Queija, I (2005). Los adolescentes españoles y su salud. Un análisis en chicos y chicas de 11 a 17 años. Madrid: Ministerio de Sanidad y Consumo.

Moreno, M.C., Muñoz, M.V., Pérez, P., Sánchez-Queija, I., Granado, M.C., Ramos, P. y Rivera, F. (2008). Desarrollo adolescente y salud. Resultados del estudio HBSC-2006 con chicos y chicas españoles de 11 a 17 años. Madrid, España: Ministerio de Sanidad y Consumo.

Moreno, M.C., Ramos, P., Rivera, F., Jiménez-Iglesias, A., García-Moya, I., Sánchez-Queija, I.,... Granado, M.C. (2012). Las conductas relacionadas con la salud y el desarrollo de los adolescentes españoles. Resultados del estudio HBSC-2010 con chicos y chicas españoles de 11 a 18 años. Madrid, España: Ministerio de Sanidad, Servicios Sociales e Igualdad.

Moreno, C., Ramos, P., Rivera, F., Jiménez-Iglesias, A., García-Moya, I., Sánchez-Queija, I.,... Morgan, A. (2016). Informe técnico de los resultados obtenidos por el Estudio Health Behaviour in School-aged Children (HBSC) 2014 en España. Madrid: Ministerio de Sanidad, Servicios Sociales e Igualdad.

Moreno, C., Ramos, P., Rivera, F., Jiménez-Iglesias, A., Garcia-Moya, I., Sánchez-Queija, I.,... Morgan, A. (en prensa). Informe técnico de los resultados obtenidos por el Estudio Health Behaviour in School-aged Children (HBSC-2018) en España. Madrid, España: Ministerio de Sanidad, Consumo y Bienestar Social.

Organisation for Economic Cooperation and Development [OECD] . (2017). Health at a Glance 2017: OECD Indicators. Paris, Francia: OECD Publishing. doi:10.1787/ health_glance-2017-18-en.

Parlamento Europeo y del Consejo. (2014). Directiva 2014/40/UE del de 3 de abril de 2014 relativa a la aproximación de las disposiciones legales, reglamentarias y administrativas de los Estados miembros en materia de fabricación, presentación y venta de los productos del tabaco y los productos relacionados. Recuperado de https://www.boe.es/ doue/2014/127/L00001-00038.pdf.

Plan Nacional sobre Drogas. (2017). Estrategia Nacional sobre Drogas 2009-2016. Informe de la Evaluación final. Madrid, España: Delegación del Gobierno para el Plan Nacional sobre Drogas, Ministerio de Sanidad, Servicios Sociales e Igualdad. Recuperado de http://www.pnsd. mscbs.gob.es/pnsd/estrategiaNacional/docs/2017_Informe_Evaluacion_Final_Estrategia_Nacional_sobre_ Drogas_2009_2016.pdf.

Plan Nacional sobre Drogas. (2018a). Encuesta sobre Alcohol y Drogas en España (EDADES) 2017. Madrid, España: Delegación del Gobierno para el Plan Nacional sobre Drogas, Ministerio de Sanidad, Servicios Sociales e Igualdad. Recuperado de http://www.pnsd.mscbs.gob. es/profesionales/sistemasInformacion/sistemaInformacion/pdf/EDADES_2017_Informe.pdf.
Plan Nacional sobre Drogas. (2018b). Encuesta sobre el uso de drogas en enseñanzas secundarias en España (ESTUDES) 2016-2017. Madrid, España: Delegación del Gobierno para el Plan Nacional sobre Drogas, Ministerio de Sanidad, Servicios Sociales e Igualdad. Recuperado de http://www.pnsd.mscbs.gob.es/profesionales/sistemasInformacion/sistemaInformacion/pdf/ESTUDES_2016_Informe.pdf.

Roberts, C., Freeman, J., Samdal, O, Schnohr, C.W., de Looze, M.E., Nic Gabhainn, S.,... Rasmussen, M. (2009). The Health Behaviour in School-aged Children (HBSC) study: methodological developments and current tensions. International Journal of Public Health, 54 (S2), 140150.

Sánchez-Queija, I., García-Moya, I y Moreno, C. (2017). Analysis of Bullying Victimization Prevalence in Spanish Adolescent Youth at School. Journal of School Health, 87, 457-464. doi:10.1111/josh.12513.

Sánchez-Queija, I., Moreno, C., Rivera, F. y Ramos, P. (2015). Tendencias en el consumo de alcohol en los adolescentes escolarizados españoles a lo largo de la primera década del siglo XXI. Gaceta Sanitaria, 29, 184-189. doi:10.1016/j.gaceta.2015.01.004.

Schnohr, C.W., Molcho, M., Rasmussen, M., Samdal, O., de Looze, M., Levin, K.,... Torsheim, T. (2015). Trend analyses in the health behaviour in school-aged children study: methodological considerations and recommendations. European Journal of Public Health, 25 (S2), 7-12.

US Department of Health and Human Services. (2012). Preventing tobacco use among youth and young adults: a report of the Surgeon General. Rockville, US: Public Health Service, Office of the Surgeon General. Recuperado de https://www.surgeongeneral.gov/library/reports/preventing-youth-tobacco-use/index.html.

Villalbí, J.R., Suelves, J.M., Saltó, E. y Cabezas, C. (2011). Valoración de las encuestas a adolescentes sobre consumo de tabaco, alcohol y cannabis en España. Adicciones, 23, 11-16. doi:10.20882/adicciones.23.1.

Villalbi, J.R., Suelves, J.M., García-Continente, X., Saltó, E., Ariza, C. y Cabezas, C. (2012). Cambios en la prevalencia del tabaquismo en los adolescentes de España. Atención Primaria, 44, 36-42. doi:10.1016/j.aprim.2010.12.016.

World Health Organization. (2003). Framework Convention on Tobacco Control. Geneva, Switzerland: WHO Press. Recuperado de http://apps.who.int/iris/bitstream/10665/42813/1/9243591010.pdf?ua=1.

World Health Organization. (2017). Tobacco, Factsheet 339. Recuperado de http://www.who.int/mediacentre/ factsheets/fs339/en/.

Zaborskis, A., Sumskas, L., Maser, M. y Pudule, I. (2006). Trends in drinking habits among adolescents in the Baltic countries over the period of transition: HBSC survey results, 1993-2002. BMC Public Health, 6, 67-77. doi:10.1186/1471-2458-6-67. 